\title{
Mappings of finite distortion: The zero set of the Jacobian
}

Received November 20, 2001 / final version received October 3, 2002

Published online November 19, 2002 - (C) Springer-Verlag \& EMS 2002

\section{Introduction}

This paper is part of our program to establish the fundamentals of the theory of mappings of finite distortion [8], [1], [10], [15], [16], [9] which form a natural generalization of the class of mappings of bounded distortion, also called quasiregular mappings. Our research continues earlier developments on mappings of finite distortion, e.g. [5], [14], [7], [18].

Let us begin with the definition. We assume that $\Omega \subset \mathbb{R}^{n}$ is a connected open set. We say that a mapping $f: \Omega \rightarrow \mathbb{R}^{n}$ has finite distortion if:

(FD-1) $f \in W_{\text {loc }}^{1,1}\left(\Omega, \mathbb{R}^{n}\right)$.

(FD-2) The Jacobian determinant $J(x, f)$ of $f$ is locally integrable.

(FD-3) There is a measurable function $K=K(x) \geq 1$, finite almost everywhere, such that $f$ satisfies the distortion inequality

$$
|D f(x)|^{n} \leq K(x) J(x, f) \quad \text { a.e. }
$$

We arrive at the usual definition of a mapping of bounded distortion (a quasiregular mapping) when we require above that $K \in L^{\infty}(\Omega)$. In this case condition (FD-2) assures that $f \in W^{1, n}\left(\Omega, \mathbb{R}^{n}\right)$. The theory of mappings of finite distortion is by now well understood, see the monographs [23] by Reshetnyak, [24] by Rickman and [13] by Iwaniec and Martin.

The recent works [3], [8], [1], [10], [15], [16], [11], [9] have established a rich theory of mappings of finite distortion under relaxed conditions on the distortion function $K$ that do not require $K$ to be bounded. Namely, it has been proven under the assumption of exponential integrability of $K$ that $f$ is continuous, sense

P. Koskela: University of Jyväskylä, Department of Mathematics and Statistics, P.O. Box 35, FIN-40351 Jyväskylä, Finland; e-mail: pkoskela@math.jyu.fi

J. Malý: Charles University, Department KMA of the Faculty of Mathematics and Physics, Sokolovská 83, CZ-18675 Praha 8, Czech Republic; e-mail: maly@karlin.mff.cuni.cz

Mathematics Subject Classification (2000): 30C65, 26B10, 73C50 
preserving, either constant or both open and discrete, and maps sets of measure zero to sets of measure zero.

The motivation for relaxing the boundedness of the distortion function partially arises from non-linear elasticity. See the paper [21] by Müller and Spector for a nice introduction to the mappings arising in that theory.

Notice that condition (FD-3) guarantees that $J(x, f) \geq 0$ a.e. in $\Omega$. However, it is not necessarily true under (FD-1) - (FD-3) that $J(x, f)>0$ a.e. in $\Omega$ for a nonconstant $f$, a condition very desirable from the point of few of the mathematical models of deformations in non-linear elasticity. It is a deep analytic fact that the Jacobian of a non-constant mapping of bounded distortion cannot vanish in a set of positive measure.

In this paper we complete the fundamentals of the theory of mappings of finite distortion by estimating the size of the zero set of the Jacobian of a mapping of unbounded finite distortion and the branch set of the mapping. Our results imply, in particular, that both the zero set of the Jacobian and the branch set of a non-constant mapping of finite, exponentially integrable distortion have volume zero. Here the branch set of $f$, denoted by $B_{f}$, consists of those points in $\Omega$ at which $f$ fails to be a local homeomorphism. We do not know of any earlier results on the size of the branch set or the zero set of the Jacobian of a mapping of a finite distortion beyond the boundedness assumption, the case of a mapping of bounded distortion.

The condition of exponential integrability of $K$ has been further relaxed in [17] as regards the topological properties of mappings of finite distortion, and our results here will also hold under these weaker assumptions. Following this work, we introduce a special class of Orlicz functions. We assume that $\Psi$ is a strictly increasing, differentiable function, and we make the following two assumptions, the second of which is entirely harmless (see the discussion in [17]):

$$
\begin{aligned}
& (\Psi-1) \int_{1}^{\infty} \frac{\Psi^{\prime}(t)}{t} d t=\infty \\
& (\Psi-2) \lim _{t \rightarrow \infty} t \Psi^{\prime}(t)=\infty
\end{aligned}
$$

Then, the right assumption on $K$ is the following condition $\left(A_{\Psi}\right)$.

$\left(A_{\Psi}\right) \exp \Psi(K)$ and $J(\cdot, f) \in L_{\text {loc }}^{1}(\Omega)$, and $\Psi$ satisfies $(\Psi-1)$ and $(\Psi-2)$.

If we know that $f \in W^{1, n}(\Omega)$, we do not need to assume so high order of integrability on $K$ to obtain the regularity results. It suffices that $K \in L_{\text {loc }}^{p}(\Omega)$ for some $p>n-1$, see [7], [18]. In this case, the requirement on the $L^{n}$-integrability of $|D f|$ can be relaxed as in [17] to:

$\left(A_{\Phi}\right) \Phi(|D f|)$ and $K^{p} \in L_{\text {loc }}^{1}(\Omega)$, where $p>n-1$ and $\Phi$ satisfies $(\Phi-1)$ and $(\Phi-2)$.

Here $\Phi$ is a strictly increasing continuous function on $[0, \infty)$ and the conditions referred to are the following:

$(\Phi-1) \int_{1}^{\infty} \frac{\Phi(t)}{t^{1+n}} d t=\infty$ 
(Ф-2) There is $q \in(n-1, n)$ such that $t \mapsto t^{-q} \Phi(t)$ increases for large values of $t$.

Under the same assumptions as considered in [17] we obtain now the results on the zero set of the Jacobian and on the branch set.

Theorem 1.1. Let $f \in W^{1,1}\left(\Omega, \mathbb{R}^{n}\right)$ satisfy the distortion inequality

$$
|D f(x)|^{n} \leq K(x) J(x, f) \quad \text { a.e. }
$$

in $\Omega$, where $K(x) \geq 1$ and either $\left(A_{\Psi}\right)$ or $\left(A_{\Phi}\right)$ is satisfied. Then $f$ (after passing to an appropriate representative) is continuous. If the Jacobian determinant of $f$ is integrable, and $f$ is not a constant mapping, then $J(x, f)>0$ a.e., $\left|B_{f}\right|=0$, and for a set $E \subset \Omega$,

$$
|f(E)|=0 \text { if and only if }|E|=0 \text {. }
$$

This result covers, for example, the case of a mapping of finite, exponentially integrable distortion. Taking Theorem 1.1 into account, we thus conclude that mappings of (sub)exponentially integrable distortion in the above sense form a natural analog of the class of mappings with bounded distortion.

In particular, Theorem 1.1 implies that, for a non-constant Sobolev mapping $f \in W^{1, n}\left(\Omega, \mathbb{R}^{n}\right)$ of distortion $K \in L^{p}(\Omega)$ for some $p>n-1$, both the branch set and set where the Jacobian vanishes are of measure zero. Thus we obtain a solution to an open problem in the paper [7] of Heinonen and Koskela; no sufficient integrability condition on the distortion was known even in this setting in space.

The original arguments of Reshetnyak [22], Martio, Rickman and Väisälä [19], Bojarski and Iwaniec [2] for the branch set and the zero set of the Jacobian in the case of mappings of bounded distortion do not seem to easily extend to our setting. It would be possible to prove Theorem 1.1 by a modification of the method of Vodop'yanov [25]. However, we use a different approach (except of Step 1 of the proof of Theorem 1.2, where the estimate is similar to a part of the argument in [25]), and prove first a result which has its own interest, Theorem 1.2.

Namely, concerning the zero set of the Jacobian, if we assume that the multiplicity of $f$ is essentially bounded, then the borderline exponent for $K$ is not $n-1$ but $n^{\prime}-1$, where $n^{\prime}=\frac{n}{n-1}$ is the conjugate exponent to $n$. In this generality, we cannot rely on any topological facilities like continuity, openness or discreteness of the mapping. The assumptions of Theorem 1.2 cover the natural and important case of mappings which are invertible in the sense that their essential multiplicity is at most one. Then the result implies, roughly speaking, that there is no cavitation under the inverse mapping.

In fact, the main role of the integrability assumptions in Theorem 1.1, which are considerably stronger than those in Theorem 1.2, is to establish a local bound on the multiplicity as the first step. This bound is obtained using the result in [18], [15] (see [17] for this generality) according to which a non-constant map satisfying the assumptions of Theorem 1.1 is both open and discrete. 
Theorem 1.2. Let $f \in W^{1,1}\left(\Omega, \mathbb{R}^{n}\right)$ satisfy the distortion inequality

$$
|D f(x)|^{n} \leq K(x) J(x, f) \quad \text { a.e. }
$$

in $\Omega$, where $1 \leq K \in L^{n^{\prime}-1}(\Omega)$. If the multiplicity of $f$ is essentially bounded by a constant $N$ and $f$ is not constant, then for any set $E \subset \Omega$ we have

$$
|f(E)|=0 \Longrightarrow|E|=0
$$

and $J(x, f)>0$ a.e. in $\Omega$.

Notice that the multiplicity is assumed to be bounded only essentially, that is, a.e. $y \in \mathbb{R}^{n}$ has at most $N$ preimages for some fixed number $N$. This means that the possibility that a preimage of a point has positive measure is not a priori excluded. As a consequence of Theorem 1.2 we obtain the "unique continuation result" that a mapping which is zero on a part of $\Omega$ of positive measure is forced to vanish on entire $\Omega$ provided it satisfies the assumptions of Theorem 1.2.

The sharpness of our results is illustrated by two examples. The first example shows that the conditions on the Orlicz classes in Theorem 1.1 cannot be violated. Also, it demonstrates that the condition on multiplicity in Theorem 1.2 cannot be dropped, even if $K$ is $L^{p}$-integrable for any $p$.

Theorem 1.3. Let $\Psi$ be an increasing, continuously differentiable function on $[0, \infty)$, not satisfying $(\Psi-1)$. Then there is a continuous, nonconstant mapping $f \in W^{1,1}\left(\mathbb{R}^{n}, \mathbb{R}^{n}\right)$ with integrable Jacobian determinant, with $f\left(\mathbb{R}^{n} \backslash B(0,1)\right)=\{0\}$ and of finite distortion with $e^{\Psi(K)}$ locally integrable.

Similarly to [17] it can be shown that such an example demonstrates also the sharpness of the condition $\left(A_{\Phi}\right)$.

The sharpness of Theorem 1.2 is demonstrated by the following theorem, which may be also obtained by considering the inverse mapping of the homeomorphism constructed in [16].

Theorem 1.4. ([13], [12]) There is a homeomorphism $f \in W^{1,1}\left(\Omega, \mathbb{R}^{n}\right)$ so that the Jacobian of $f$ vanishes in a set of positive volume and $f$ has a distortion that belongs to $L_{l o c}^{p}(\Omega)$ for all $p<n^{\prime}-1$.

\section{Proofs of Theorems 1.2 and 1.1}

We need the following consequence of standard covering arguments.

Lemma 2.1. There is a constant $\tau=\tau(n)$ with the following property: For each atomless probability Borel measure $\mu$ on $\mathbb{R}^{n}$ there is a point $y \in \mathbb{R}^{n}$ and a radius $R>0$ such that

$$
\mu(B(y, 2 R)) \geq \tau \quad \text { and } \quad \mu\left(\mathbb{R}^{n} \backslash B(y, 3 R)\right) \geq \tau .
$$


Proof. There is a constant $L$ depending only on $n$ such that any ball with radius $3 r$ can be covered by $L$ balls with radius $r$. Set

$$
\tau=\frac{1}{L+1}
$$

Let

$$
\rho=\inf \left\{r>0:\left[\exists y \in \mathbb{R}^{n}: \mu(B(y, r)) \geq \tau\right]\right\} .
$$

Since $\mu$ does not have atoms and $\mu\left(\mathbb{R}^{n}\right)<\infty$, it easily follows that $\rho>0$. We find a point $y \in \mathbb{R}^{n}$ and a radius $R>0$ such that

$$
\mu(B(y, 2 R)) \geq \tau \quad \text { and } \quad R<\rho<2 R .
$$

Consider a collection $B_{1}, \ldots, B_{L}$ of balls with radii $R$ that cover $B(y, 3 R)$. Since $R<\rho$, we have

$$
\mu\left(B_{i}\right) \leq \tau, \quad i=1, \ldots, L
$$

and thus

$$
\mu(B(y, 3 R)) \leq \sum_{i=1}^{L} \mu\left(B_{i}\right) \leq L \tau,
$$

which means, since $\mu\left(\mathbb{R}^{n}\right)=1$,

$$
\mu\left(\mathbb{R}^{n} \backslash B(y, 3 R)\right) \geq 1-L \tau=\tau .
$$

Proof of Theorem 1.2. Let us shortly write $J=J(\cdot, f)$, The proof will be divided into four steps.

STEP 1. We first prove an auxiliary estimate. Let $E \subset \Omega$ be a measurable set. Consider a smooth function $u$ with a compact support in $\mathbb{R}^{n}$. A direct computation gives

$$
\begin{aligned}
& \int_{E}|\nabla(u \circ f)| d x \\
& \leq \int_{E}|(\nabla u) \circ f||D f| d x \\
& \leq \int_{E}|(\nabla u) \circ f| J^{1 / n} K^{1 / n} d x \\
& \leq\left(\int_{E}|(\nabla u) \circ f|^{n} J d x\right)^{1 / n}\left(\int_{E} K^{n^{\prime}-1} d x\right)^{1 / n^{\prime}} \\
& \leq\left(N \int_{\mathbb{R}^{n}}|\nabla u|^{n} d x\right)^{1 / n}\left(\int_{E} K^{n^{\prime}-1} d x\right)^{1 / n^{\prime}} .
\end{aligned}
$$

Here we used the fact that for a mapping $f \in W^{1,1}\left(\Omega ; \mathbb{R}^{n}\right)$ of multiplicity essentially bounded by $N$ and a nonnegative Borel measurable function $h$ on $\mathbb{R}^{n}$,

$$
\int_{\Omega} h(f(x)) J(x, f) d x \leq N \int_{\mathbb{R}^{n}} h(y) d y,
$$


that follows from the area formula and the fact (proved in [4]) that $\Omega$ can be exhausted up to a set of measure zero by sets the restriction to which of $f$ is Lipschitz continuous. See also [6].

STEP 2. We claim that

$$
y_{0} \in \mathbb{R}^{n} \Longrightarrow\left|f^{-1}\left(\left\{y_{0}\right\}\right)\right|=0 \text {. }
$$

For this, consider an arbitrary ball $B \subset \subset \Omega$ and $y_{0} \in \mathbb{R}^{n}$. Suppose that $f$ differs from $y_{0}$ on a set of positive measure in $B$. Then there is $R>0$ such that

$$
\kappa:=\left|B \backslash f^{-1}\left(B\left(y_{0}, R\right)\right)\right|>0 .
$$

Since singletons have zero $n$-capacity, given $\varepsilon>0$ there is a smooth function $u$ on $\mathbb{R}^{n}$ such that

$$
\text { spt } u \in B\left(y_{0}, R\right), \quad u(0)=1 \quad \text { and } \quad \int_{\mathbb{R}^{n}}|\nabla u|^{n} d y<\varepsilon^{n} .
$$

Then

$$
\min \left\{\left|B \cap f^{-1}\left(y_{0}\right)\right|, \kappa\right\} \leq C r \int_{B}|\nabla(u \circ f)| d x .
$$

For this we used the well-known trick

$$
\frac{1}{2} \min \{|B \cap\{v \leq 0\}|,|B \cap\{v \geq 1\}|\} \leq \inf _{c \in \mathbb{R}} \int_{B}|v-c| d x \leq C r \int_{B}|\nabla v| d x,
$$

based on the Poincaré inequality, where the hypothesis is that $v \in W^{1,1}(B)$. By (2.1), (2.5) and (2.6) we have

$$
\begin{aligned}
\min \left\{\left|B \cap f^{-1}\left(y_{0}\right)\right|, \kappa\right\} & \leq C r\left(N \int_{\mathbb{R}^{n}}|\nabla u|^{n} d x\right)^{1 / n}\left(\int_{B} K^{n^{\prime}-1} d x\right)^{1 / n^{\prime}} \\
& \leq C \varepsilon\left(\int_{B} K^{n^{\prime}-1} d x\right)^{1 / n^{\prime}} .
\end{aligned}
$$

Letting $\varepsilon \rightarrow 0$ and using (2.4) we obtain that $\left|B \cap f^{-1}\left(y_{0}\right)\right|=0$ whenever $f$ differs from $y_{0}$ on a set of positive measure in $B$. Hence (2.3) follows by taking the connectedness of $\Omega$ and the assumption that $f$ is not constant into account.

STEP 3. Now, let us prove that $J>0$ a.e. Denote by $Z$ the zero set of the Jacobian. Fix a ball $B\left(x_{0}, r\right) \subset \Omega$. Consider the Borel measure defined by

$$
\mu(A)=\frac{\left|B\left(x_{0}, r\right) \cap f^{-1}(A)\right|}{\left|B\left(x_{0}, r\right)\right|}, \quad A \subset \mathbb{R}^{n} .
$$

By (2.3), $\mu$ does not have atoms. Using Lemma 2.1 we find a point $y \in \mathbb{R}^{n}$ and a radius $R>0$ such that

$$
\mu(B(y, 2 R)) \geq \tau \quad \text { and } \quad \mu\left(\mathbb{R}^{n} \backslash B(y, 3 R)\right) \geq \tau .
$$


where $\tau=\tau(n)>0$. Let $u$ be a smooth function on $\mathbb{R}^{n}$ such that

$$
\text { spt } u \subset B(y, 3 R), \quad u=1 \text { in } B(y, 2 R), \quad \int_{\mathbb{R}^{n}}|\nabla u|^{n} d y \leq C(n),
$$

and set

$$
v:=u \circ f .
$$

Then $v \in W^{1,1}\left(B\left(x_{0}, r\right)\right)$ and by (2.8) and (2.9),

$$
\begin{gathered}
\frac{\mid B\left(x_{0}, r\right) \cap\{v=1\}}{\left|B\left(x_{0}, r\right)\right|}=\frac{\left.\mid B\left(x_{0}, r\right) \cap f^{-1}(B(y, 2 R))\right\} \mid}{\left|B\left(x_{0}, r\right)\right|}=\mu(B(y, 2 R)) \geq \tau, \\
\frac{\left|B\left(x_{0}, r\right) \cap\{v=0\}\right|}{\left|B\left(x_{0}, r\right)\right|} \geq \frac{\left.\mid B\left(x_{0}, r\right) \backslash f^{-1}(B(y, 3 R))\right\} \mid}{\left|B\left(x_{0}, r\right)\right|}=\mu\left(\mathbb{R}^{n} \backslash B(y, 3 R)\right) \geq \tau .
\end{gathered}
$$

By the Poincaré inequality (2.7) we have

$$
\begin{aligned}
1 & \leq C r^{-n} \inf _{c \in \mathbb{R}} \int_{B\left(x_{0}, r\right)}|v-c| d x \leq C r^{1-n} \int_{B\left(x_{0}, r\right)}|\nabla v| d x \\
& =C r^{1-n} \int_{B\left(x_{0}, r\right)}|\nabla(u \circ f)| d x .
\end{aligned}
$$

Since $f$ is a mapping of bounded distortion, we have $D f=0$ and thus $\nabla(u \circ f)=0$ a.e. on $Z$. Hence by (2.11), (2.1) and (2.9)

$$
1 \leq C r^{1-n} \int_{B\left(x_{0}, r\right) \backslash Z}|\nabla(u \circ f)| d x \leq C\left(r^{-n} \int_{B\left(x_{0}, r\right) \backslash Z} K^{n^{\prime}-1}\right)^{1 / n^{\prime}} .
$$

If $x_{0}$ is a Lebesgue point for $g:=K^{n^{\prime}-1} \chi_{\Omega \backslash Z}$, where $\chi_{\Omega \backslash Z}$ is the characteristic function of $\Omega \backslash Z$, it follows that $g\left(x_{0}\right)>0$. This means that the set $Z$ does not contain any Lebesgue points for $g$, and so it must be of measure zero. We have proved that $J>0$ a.e.

STEP 4. Finally, we will prove (1.1). Given $E \subset \Omega$ with $|f(E)|=0$, we find a Borel measurable set $A \subset \mathbb{R}^{n}$ of measure zero which contains $|f(E)|$. Then $E$ is contained in the measurable set $E^{\prime}=f^{-1}(A)$. Let $h$ be the characteristic function of $A$. By (2.2) we have

$$
\int_{E^{\prime}} J(x, f) d x=\int_{\Omega} h(f(x)) J(x, f) d x \leq N \int_{\mathbb{R}^{n}} h(y) d y=0 .
$$

Since $J>0$ a.e. it follows that $|E|=0$.

Proof of Theorem 1.1. By the results in [10], [15] and [17], $f$ is continuous and both open and discrete. In particular, the multiplicity of $f$ is locally bounded. Thus we may apply Theorem 1.2 to conclude that $J(x, f)>0$ a.e.

Regarding the size of the branch set $B_{f}$, recall from [8], [10], [17] that $f$ is monotone and belongs to $W^{1, p}\left(\Omega, \mathbb{R}^{n}\right)$ for all $p<n$. Thus, by Väisälä's theorem on differentiability of monotone Sobolev mappings (cf. [24, Lemma 4.4]), $f$ is 
differentiable a.e. in $\Omega$. Taking the a.e. strict positivity of the Jacobian into account, we conclude that, for a.e. $x$ in $\Omega, f$ is differentiable with non-zero Jacobian. It follows that the branch set has measure zero, see e.g. [24, Lemma 4.12].

The last part of the claim of Theorem 1.1 follows from (1.1) when we combine this with the result from [17], according to which $f$ maps sets of measure zero to sets of measure zero.

\section{Proof of Theorem 1.3}

We begin the construction of the example referred to in Theorem 1.3 by recalling the existence of suitable auxiliary Lipschitz functions.

Lemma 3.1. Let $\tau>0$ and $1 \leq i \leq n$. Then there is a Lipschitz function $u$ : $Q_{0} \rightarrow \mathbb{R}$ such that $u=0$ on the boundary of the unit cube $Q_{0}$, the alternative

$$
\left|\nabla u(x)-\mathbf{e}_{i}\right|<\tau \text { or }\left|\nabla u(x)+\mathbf{e}_{i}\right|<\tau
$$

holds for a.e. $x \in Q_{0}$, and $u$ is smooth outside a closed set of measure zero.

Proof. See the work of Fonseca, Müller and Šverák, [20, Lemma 5.1] and references therein.

Proof of Theorem 1.3. Our aim is to construct a mapping $f: Q_{0} \rightarrow \mathbb{R}^{n}$ so that $f$ is of finite distortion $K, f=0$ on $\partial Q_{0}$ and the distortion of $f$ is as small as possible. To obtain the mapping referred to in Theorem 1.3, we then simply extend $f$ as the constant mapping zero to the rest of $\mathbb{R}^{n}$ and scale the variable to guarantee that $f$ maps the exterior of the unit ball to the origin; the rest of the properties will follow from our construction.

The plan is to first to build a Lipschitz mapping with the correct boundary values using Lemma 3.1. This mapping will have bounded distortion in the set where the Jacobian is positive. We will then modify our mapping in the set where its Jacobian is negative by precomposing it (locally) with suitable mappings with negative Jacobians. For this we will use our construction in [15] and its refinement in [17].

Given $\tau>0$, using Lemma 3.1 we construct a mapping $u=\left(u_{1}, \ldots, u_{n}\right)$ : $Q_{0} \rightarrow \mathbb{R}^{n}$ so that for $i=1, \ldots, n$ and a.e. $x \in Q_{0}$ we have

$$
\left|\nabla u_{i}(x)-\mathbf{e}_{i}\right|<\tau \text { or }\left|\nabla u_{i}(x)+\mathbf{e}_{i}\right|<\tau .
$$

Let $\Omega \subset Q_{0}$ be an open set of full measure where $u$ is smooth. Set

$$
\Omega^{+}=\{x \in \Omega: J(x, u)>0\}, \quad \Omega^{-}=\{x \in \Omega: J(x, u)<0\} .
$$

By choosing a suitably small $\tau$ we may assume that

$$
|D u| \leq 2
$$


and that a.e. in $Q_{0}$ either

$$
1 / 2 \leq J(x, u) \leq 3 / 2
$$

or

$$
-3 / 2 \leq J(x, u) \leq-1 / 2 .
$$

Then on $\Omega^{+}$we have, by (3.1), (3.2),

$$
|D u(x)|^{n} \leq 2^{n+1} J(x, u) .
$$

We define

$$
f(x)=u(x), \quad x \notin \Omega^{-} .
$$

On $\Omega^{-}$, we will precompose $u$ with suitable mappings with negative Jacobians so as to obtain a mapping with positive Jacobian. For this we recall the mapping constructed in [17] as a refinement of the example in [15]: under the assumptions of Theorem 1.3 there is a continuous mapping $g \in W^{1,1}\left(Q_{0}, M Q_{0}\right)$ for some $M>1$ so that $g(x)=x$ on the boundary of the unit cube $Q_{0}, J(x, g)<0$ a.e. in $Q_{0}$,

$$
\int_{Q_{0}}|J(x, g)| d x=a<\infty
$$

and $g$ satisfies

$$
|D g(x)|^{n} \leq K(x)|J(x, g)|
$$

a.e. with

$$
\int_{Q_{0}} e^{\Psi\left(2^{n+1} K\right)} d x=b<\infty .
$$

We will now use several scaled and translated copies of $g$ attached to cubes in $\Omega^{-}$. Instead of the usual Whitney decomposition of $\Omega^{-}$, we pick a finer, similar decomposition $\mathcal{Q}$ of $\Omega^{-}$into cubes $Q$ so that $M \bar{Q} \subset \Omega^{-}$for each cube in this Whitney decomposition $\mathcal{Q}$. Using the above mapping $g$ we find, using translations and scaling, for each $Q \in \mathcal{Q}$, a mapping

$$
g_{Q}: \bar{Q} \rightarrow M \bar{Q}
$$

such that $g_{Q}(x)=x$ on $\partial Q, J\left(x, g_{Q}\right)<0$ a.e.,

$$
\begin{gathered}
\int_{Q}\left|J\left(x, g_{Q}\right)\right| d x \leq a|Q|, \\
\int_{Q}\left|D g_{Q}\right| d x \leq c|Q|,
\end{gathered}
$$

and

$$
\left|D g_{Q}(x)\right|^{n} \leq K(x)\left|J\left(x, g_{Q}\right)\right|
$$


a.e. with

$$
\int_{Q} e^{\Psi\left(2^{n+1} K\right)} d x \leq b|Q| .
$$

We define

$$
f(x)=u\left(g_{Q}(x)\right), \quad x \in \bar{Q}
$$

when $Q \in \mathcal{Q}$. Then (3.4) and (3.8) complete the construction because $\Omega^{-}=$ $\cup_{Q \in \mathcal{Q}} Q$.

To see that $f$ has the desired properties, notice first that the distortion of $f$ on $Q$ is, by (3.1), (3.3), no more than $2^{n+1}$ times the "distortion" of $g_{Q}$ on $Q$. The desired integrability condition on the distortion of $f$ then follows using (3.7). The construction shows that $f \in W^{1,1}\left(Q_{0}, \mathbb{R}^{n}\right)$ and that $f$ is continuous. Finally, the integrability of the Jacobian of $f$ follows from the definition of $f$ and from (3.2), (3.3), and (3.5).

Acknowledgements. Pekka Koskela was supported in part by the Academy of Finland, project 39788. Jan Malý is supported by the Research Project MSM 113200007 from the Czech Ministry of Education, Grant No. 201/00/0767 from the Grant Agency of the Czech republic (GA R) and Grant No. 165/99 from the Grant Agency of Charles University (GA UK).

\section{References}

1. Astala, K., Iwaniec, T., Koskela, P., Martin, G.: Mappings of BMO-bounded distortion. Math. Ann. 317, 703-726 (2000)

2. Bojarski, B., Iwaniec, T.: Analytical foundations of the theory of quasiconformal mappings in $R^{n}$. Ann. Acad. Sci. Fenn., Ser. A I, Math. 8, 257-324 (1983)

3. David, G.: Solutions de l'equation de Beltrami avec $\|\mu\|_{\infty}=1$. Ann Acad. Sci. Fenn., Ser. A I, Math. 13, 25-70 (1988)

4. Federer, H.: Surface area II. Trans. Am. Math. Soc. 55, 438-456 (1944)

5. Gol'dstein, V., Vodop'yanov, S.: Quasiconformal mappings and spaces of functions with generalized first derivatives. Sib. Mat. Zh. 17, 515-531 (1976)

6. Hajłasz, P.: Change of variables formula under minimal assumptions. Colloq. Math. 64, 93-101 (1993)

7. Heinonen, J., Koskela, P.: Sobolev mappings with integrable dilatations. Arch. Ration. Mech. Anal. 125, 81-97 (1993)

8. Iwaniec, T., Koskela P., Martin, G.: Mappings of BMO-distortion and Beltrami type operators. J. Anal. Math. To appear

9. Iwaniec, T., Koskela P., Martin, G., Sbordone, C.: Mappings of exponentially integrable distortion. J. London Math. Soc., II. Ser. To appear

10. Iwaniec, T., Koskela, P., Onninen, J.: Mappings of finite distortion: Monotonicity and continuity. Invent. Math. 144, 507-531 (2001)

11. Iwaniec, T., Martin, G.: Beltrami equations. Mem. Am. Math. Soc. To appear

12. Iwaniec, T., Martin, G.: Squeezing the Sierpinski sponge. Stud. Math. 149, 133-145 (2002)

13. Iwaniec, T., Martin, G.: Geometric Function Theory and Nonlinear Analysis. Oxford Univ. Press. 2001

14. Iwaniec, T., Šverák, V.: On mappings with integrable dilatation. Proc. Am. Math. Soc. 118, 181-188 (1993) 
15. Kauhanen, J., Koskela, P., Malý, J.: Mappings of finite distortion: Discreteness and openness. Arch. Ration. Mech. Anal. 160, 135-151 (2001)

16. Kauhanen, J., Koskela, P., Malý, J.: Mappings of finite distortion: Condition N. Mich. Math. J. 49, 169-181 (2001)

17. Kauhanen, J., Koskela, P., Malý, J., Onninen, J., Zhong, X.: Mappings of finite distortion: Sharp Orlicz-conditions. Preprint 239, Department of Math., University of Jyväskylä 2001

18. Manfredi, J., Villamor, E.: Mappings with integrable dilatation in higher dimensions. Bull. Am. Math. Soc., New Ser. 32, 235-240 (1995)

19. Martio, O., Rickman, S., Väisälä, J.: Definitions for quasiregular mappings. Ann. Acad. Sci. Fenn., Ser. A I, Math. 448, 1-40 (1969)

20. Müller, S.: Variational models for microstructure and phase transitions. Calculus of variations and geometric evolution problems (Cetraro, 1996), 85-210, Lecture Notes in Math., 1713, Springer, Berlin 1999

21. Müller, S., Spector, S.: An existence theory for nonlinear elasticity that allows for cavitation. Arch. Ration. Mech. Anal. 131, 1-66 (1995)

22. Reshetnyak, Yu.G.: Space Mappings with Bounded Distortion. Sib. Mat. Zh. 8, 629-658 (1967)

23. Reshetnyak, Yu.G.: Space Mappings with Bounded Distortion. Trans. of Mathematical Monographs, vol. 73, Am. Math. Soc. 1989

24. Rickman, S.: Quasiregular mappings. Ergebnisse der Mathematik und ihrer Grenzgebiete (3) [Results in Mathematics and Related Areas (3)], 26. Springer-Verlag, Berlin 1993

25. Vodop'yanov, S.: Mappings with bounded distortion and with finite distortion on Carnot groups. Sib. Math. J. 40, 764-804 (1999) 